\title{
Multiple critical endpoints in magnetized three flavor quark matter
}

\author{
Márcio Ferreira, ${ }^{*}$ Pedro Costa, ${ }^{\dagger}$ and Constança Providência ${ }^{*}$ \\ CFisUC, Department of Physics, University of Coimbra, P-3004-516 Coimbra, Portugal
}

(Received 20 November 2017; published 30 January 2018)

\begin{abstract}
The magnetized phase diagram for three-flavor quark matter is studied within the Polyakov extended Nambu-Jona-Lasinio model. The order parameters are analyzed with special emphasis on the strange quark condensate. We show that the presence of an external magnetic field induces several critical endpoints (CEPs) in the strange sector, which arise due to the multiple phase transitions that the strange quark undergoes. The spinodal and binodal regions of the phase transitions are shown to increase with external magnetic field strength. The influence of strong magnetic fields on the isentropic trajectories around the several CEPs is analyzed. A focusing effect is observed on the region towards the CEPs that are related with the strange quark phase transitions. Compared to the chiral transitions, the deconfinement transition turns out to be less sensitive to the external magnetic field and the crossover nature is preserved over the whole phase diagram.
\end{abstract}

DOI: 10.1103/PhysRevD.97.014014

\section{INTRODUCTION}

The possible existence of a critical endpoint (CEP) in the QCD phase diagram is a long-standing issue that has captured the attention of the physics community. The nature of the phase transition between hadron matter and quark gluon plasma (QGP) can be inferred from its existence. A wide range of theoretical frameworks have been applied in analyzing the QCD phase diagram and the possible existence of a CEP: lattice QCD (LQCD) simulations [1,2]; Dyson-Schwinger equations [3]; and several effective models, namely, the Nambu-Jona-Lasinio (NJL) model [4], its extension up to eight-quark terms [5], and the Polyakov-Nambu-Jona-Lasinio (PNJL) model [6].

The QGP expansion is recognized as a hydrodynamic expansion of an ideal fluid, which follows trajectories of constant entropy, known as isentropes. The conservation of the baryon number restricts the isentropic trajectories to lines of constant entropy per baryon $\left(s / \rho_{B}\right)$ in the $\left(T, \mu_{B}\right)$ space with zero strange quark density. These isentropic trajectories contain important information on the adiabatic evolution of the system. For AGS, SPS, and RHIC, the values of $s / \rho_{B}$ are 30,45, and 300, respectively [7]. Lattice results for the $(2+1)$-flavor equation of state $(\mathrm{EOS})$ at these $s / \rho_{B}$ values are given in Refs. [8,9]. The presence of

\footnotetext{
*mferreira@teor.fis.uc.pt

pcosta@uc.pt

*cp@fis.uc.pt
}

Published by the American Physical Society under the terms of the Creative Commons Attribution 4.0 International license. Further distribution of this work must maintain attribution to the author(s) and the published article's title, journal citation, and DOI. Funded by SCOAP. a CEP in the QCD phase diagram might deform the isentropes' trajectories [10]. This reinforces the importance of the search for the CEP, because modifications of the expansion trajectory may lead to observable effects in the hadron spectra (see Ref. [11]).

From the experimental point of view, the CEP existence/ location is a major goal of several heavy-ion collision (HIC) programs. The Beam Energy Scan (BES-I) program at the RHIC searched for experimental signatures of the CEP, by colliding Au ions at several energies [12]. Results of the moments of net-charge multiplicity distributions [13] and of moments of net-kaon (proxy for netstrangeness) multiplicity distributions [14] from the STAR Collaboration provide relevant information on the freezeout conditions (also for strange quarks) and can help to clarify the existence of the CEP. However, high precision measurements and high statistics data are required for definitive conclusions regarding the CEP and precise determination of the freeze-out conditions $[13,14]$. High precision measurements for the net-kaon fluctuations will be made in the second phase of the RHIC BES. Furthermore, QCD calculations should take into account the dynamics associated with heavy-ion collisions before definitive conclusions about the CEP can be made [15]. The results from the NA61/SHINE experiment at CERN SPS on the particle spectra and fluctuations (in $\mathrm{p}+\mathrm{p}, \mathrm{Be}+\mathrm{Be}$, and $\mathrm{Ar}+\mathrm{Sc}$ collisions) show, so far, no indications of the existence of a CEP $[16,17]$. In the near future, planned experiments at FAIR (GSI) and NICA (JINR) will extend the search for a first-order phase transition and the CEP to regions of higher baryonic chemical potentials and lower temperatures, and hopefully unveil the existence and location of the CEP on the QCD phase diagram (see [18] for a review on the experimental search of the CEP). 
Strong external magnetic fields may play a role in multiple physical systems: from HIC experiments at very high energies, to the early stages of the Universe, and astrophysical objects like magnetized neutron stars. It becomes crucial, therefore, to understand how an external magnetic field affects the structure of the QCD phase diagram. Several LQCD calculations have been performed that address the impact of the magnetic field over the deconfinement and chiral transitions [19-24]. Besides the catalyzing effect of $B$ on dynamical chiral symmetry breaking, known as the magnetic catalysis (MC) effect (see [25] for a review), LQCD results show also an additional effect: in the crossover transition region, the magnetic field, instead of catalyzing, weakens the dynamical chiral symmetry breaking [19-21]. This additional phenomenon is called the inverse magnetic catalysis (IMC) effect (for a review, see [26]). Several low-energy effective models, including the NJL-type models, have been used to investigate the MC effect and its impact at finite chemical potential [27-39].

The existence/location of the CEP can be influenced by the medium strangeness and isospin content, and by the presence and strength of an external magnetic field [40]. Within the $(2+1)$-NJL model, it was verified that the CEP becomes located at higher temperatures with increasing $B$ [41]. The same was obtained within the Ginzburg-Landau effective action formalism with the renormalized quarkmeson model [42]. Using the $(2+1)$-PNJL model, the role played by vector interactions and the IMC effect on the CEP's location was analyzed in [43], where opposite competing effects were found. Another interesting aspect of the QCD phase diagram is the possible existence of a $\mathrm{CEP}$ associated with the strange quarks (with the respective first-order phase transition) in a generalized NJL model with the inclusion of explicit chiral symmetry breaking interactions [5]. This implies the existence of two CEPs in the phase diagram. Indeed, the presence of these interactions acts as a catalyst in the production of strange quark matter when compared to conventional versions of the NJL model [5]. Thus, it is interesting to explore in detail the effect of external magnetic fields on the strange sector, looking for the possible emergence of a CEP in this sector due to the catalyzing effect of $B$ at lower temperatures.

In the present work, we investigate the magnetized phase diagram using the $(2+1)$-flavor PNJL model. Special attention is devoted to the strange quark phase transition and the CEPs that appear in the presence of an external magnetic field. We investigate the isentropic trajectories near the light and strange CEPs, in both the crossover and first-order transition regions. The model is presented in Sec. II, while the results are in Sec. III. Finally we draw our conclusions in Sec. IV.

\section{MODEL AND FORMALISM}

In the presence of an external magnetic field, the Lagrangian density of the PNJL model for a $(2+1)$-flavor field takes the following form,

$$
\begin{aligned}
\mathcal{L}= & \bar{q}\left[i \gamma_{\mu} D^{\mu}-\hat{m}_{c}\right] q+\mathcal{L}_{\mathrm{sym}}+\mathcal{L}_{\mathrm{det}}-\frac{1}{4} F_{\mu \nu} F^{\mu \nu} \\
& +\mathcal{U}(\Phi, \bar{\Phi} ; T)
\end{aligned}
$$

where the quark sector is described by the $\mathrm{SU}(3)$ version of the NJL model [44,45], which includes scalar-pseudoscalar, $\mathcal{L}_{\text {sym }}$, and the 't Hooft six-fermion interaction, $\mathcal{L}_{\text {det }}$,

$$
\begin{aligned}
\mathcal{L}_{\text {sym }} & =G_{s} \sum_{a=0}^{8}\left[\left(\bar{q} \lambda_{a} q\right)^{2}+\left(\bar{q} i \gamma_{5} \lambda_{a} q\right)^{2}\right] \\
\mathcal{L}_{\text {det }} & =-K\left\{\operatorname{det}\left[\bar{q}\left(1+\gamma_{5}\right) q\right]+\operatorname{det}\left[\bar{q}\left(1-\gamma_{5}\right) q\right]\right\} .
\end{aligned}
$$

The quark field is represented in flavor space by $q=(u, d, s)^{T}$, and $\hat{m}_{c}=\operatorname{diag}_{f}\left(m_{u}, m_{d}, m_{s}\right)$ is the corresponding (current) mass matrix. The Gell-Mann matrices are denoted by $\lambda_{a}(0<a \leq 8)$ and $\lambda_{0}=\sqrt{2 / 3} I$, where $I$ represents the unit matrix. The coupling between the (electro)magnetic field $B$ and both the quarks and the effective gluon field is implemented via the covariant derivative $D^{\mu}=\partial^{\mu}-i q_{f} A_{\mathrm{EM}}^{\mu}-i A^{\mu}$, where $q_{f}$ represents the quark electric charge $\left(q_{d}=q_{s}=-q_{u} / 2=-e / 3\right) ; A_{\mathrm{EM}}^{\mu}$ is the external magnetic field $\left(F^{\mu \nu}=\partial^{\mu} A_{\mathrm{EM}}^{\nu}-\partial^{\nu} A_{\mathrm{EM}}^{\mu}\right)$; and $A^{\mu}(x)=g_{\text {strong }} \mathcal{A}_{a}^{\mu}(x) \frac{\lambda_{a}}{2}$, where $\mathcal{A}_{a}^{\mu}$ is the $\mathrm{SU}_{c}(3)$ gauge field. We consider a static and constant magnetic field in the $z$ direction, $A_{\mathrm{EM}}^{\mu}=\delta^{\mu 2} x_{1} B$. The spatial components of the gluon field are neglected in the Polyakov gauge at finite temperature, $A^{\mu}=\delta_{0}^{\mu} A^{0}=-i \delta_{4}^{\mu} A^{4}$. The Polyakov loop is defined as the trace of the Polyakov line, $\Phi=$ $\frac{1}{N_{c}}\left\langle\left\langle\mathcal{P} \exp i \int_{0}^{\beta} d \tau A_{4}(\vec{x}, \tau)\right\rangle\right\rangle_{\beta}$, which is the order parameter of the $\mathbb{Z}_{3}$ symmetric/broken phase transition in pure gauge.

The pure gauge sector is described by the following effective potential $\mathcal{U}(\Phi, \bar{\Phi} ; T)[46]$,

$$
\begin{aligned}
\frac{\mathcal{U}(\Phi, \bar{\Phi} ; T)}{T^{4}}= & -\frac{a(T)}{2} \bar{\Phi} \Phi \\
& +b(T) \ln \left[1-6 \bar{\Phi} \Phi+4\left(\bar{\Phi}^{3}+\Phi^{3}\right)-3(\bar{\Phi} \Phi)^{2}\right],
\end{aligned}
$$

where $a(T)=a_{0}+a_{1}\left(\frac{T_{0}}{T}\right)+a_{2}\left(\frac{T_{0}}{T}\right)^{2}, b(T)=b_{3}\left(\frac{T_{0}}{T}\right)^{3}$. The parameters were fitted to reproduce lattice results [46]: $a_{0}=3.51, a_{1}=-2.47, a_{2}=15.2$, and $b_{3}=-1.75$. The critical temperature for the deconfinement phase transition is set by the parameter $T_{0}$, which in pure gauge was fixed to $T_{0}=270 \mathrm{MeV}$.

As a regularization scheme, we use a sharp cutoff $\Lambda$ in three-momentum space for the divergent ultraviolet sea quark integrals. For the model parametrization, we consider [47] $\Lambda=602.3 \mathrm{MeV}, \quad m_{u}=m_{d}=5.5 \mathrm{MeV}$, $m_{s}=140.7 \mathrm{MeV}, G_{s}^{0} \Lambda^{2}=1.835$, and $K \Lambda^{5}=12.36$.

In the present study, we consider two model variants with distinct scalar couplings: constant $G_{s}=G_{s}^{0}$ and a magnetic 
field dependent $G_{s}=G_{s}(e B)$ [31]. In the latter case, the magnetic field dependence was determined phenomenologically, by reproducing the decrease in ratio of the chiral pseudocritical temperature obtained in LQCD calculations [20]. Its functional dependence is $G_{s}(\zeta)=G_{s}^{0}\left(\frac{1+a \zeta^{2}+b \zeta^{3}}{1+c \zeta^{2}+d \zeta^{4}}\right)$, where $\zeta=e B / \Lambda_{\mathrm{QCD}}^{2} \quad$ (with $\Lambda_{\mathrm{QCD}}=300 \mathrm{MeV}$ ). The parameters are $a=0.0108805, b=-1.0133 \times 10^{-4}$, $c=0.02228$, and $d=1.84558 \times 10^{-4}$ [31]. At zero magnetic field both models coincide, i.e., $G_{s}=G_{s}^{0}=$ $G_{s}(e B=0)$.

For each value of temperature $T$, baryonic chemical potential $\mu_{B}$, and magnetic field strength $B$, the mean field equations are obtained by minimizing the thermodynamic potential with respect to the order parameters [48]: $\langle u \bar{u}\rangle$, $\langle d \bar{d}\rangle,\langle s \bar{s}\rangle, \Phi$, and $\bar{\Phi}$. Both the chiral and the deconfinement phase transitions can show different natures: first-order, second-order, or crossover (analytic transition). Contrarily to first-order phase transitions, the crossover transition is characterized by an analytic behavior, allowing for different definitions of (pseudo)critical temperature through different observables. The pseudocritical temperature is often defined as the temperature at which the inflection point of the order parameters occurs. Nevertheless, another possible definition is the temperature at which the order parameter reaches half its vacuum value, i.e., $\langle q \bar{q}\rangle\left(T, \mu_{B}\right) /\langle q \bar{q}\rangle(0,0)=0.5$ for quarks, and $\Phi\left(T, \mu_{B}\right)=0.5$ for the Polyakov loop. As we are going to analyze the order parameters via contour diagrams, the latter definition of pseudocritical temperature will be useful.

\section{RESULTS}

Herein, we consider the PNJL model with equal quark chemical potentials, $\mu_{u}=\mu_{d}=\mu_{s}=\mu_{q}$, which corresponds to zero charge (or isospin) chemical potential and zero strangeness chemical potential, i.e., $\mu_{Q}=\mu_{S}=0$. The baryonic chemical potential is then given by $\mu_{B}=3 \mu_{q}$.

\section{A. Magnetized phase diagram}

To analyze how an external magnetic field affects the chiral/deconfinement transitions, we determine the quark condensates and the Polyakov loop value (order parameters) in the $\left(T, \mu_{B}\right)$ plane, for two magnetic field strengths: $e B=0.3 \mathrm{GeV}^{2}$ and $e B=0.6 \mathrm{GeV}^{2}$. As we are mainly interested in examining where the phase transitions occur rather than on the specific condensates values, we normalize the condensates as

$$
\langle q \bar{q}\rangle_{0}=\langle q \bar{q}\rangle_{0}\left(T, \mu_{B}, e B\right)=\frac{\langle q \bar{q}\rangle\left(T, \mu_{B}, e B\right)}{\langle q \bar{q}\rangle(0,0, e B)} .
$$

This way, regardless of the magnetic field strength, the normalized condensate $\langle q \bar{q}\rangle_{0}$ lies between 0 and 1 . If one
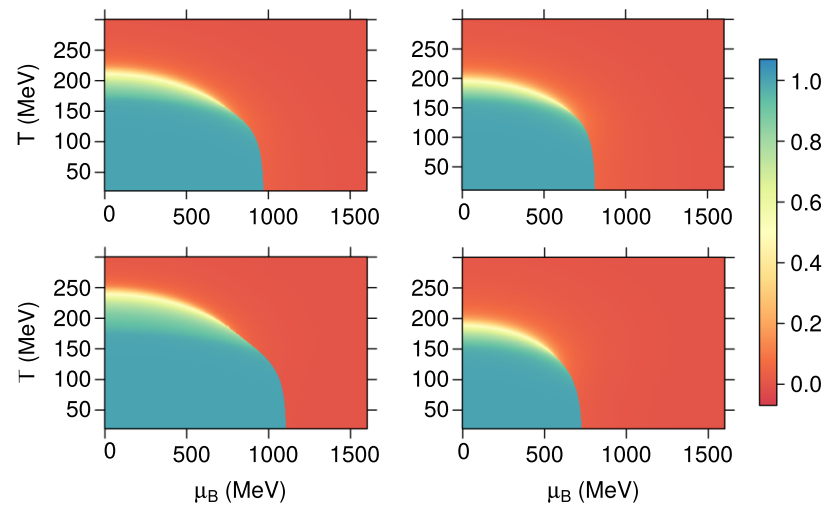

FIG. 1. The normalized up-quark condensate $\langle u \bar{u}\rangle_{0}$ (the color scale represents its magnitude) with $G_{s}^{0}$ (left) and $G_{s}(e B)$ (right) for $e B=0.3 \mathrm{GeV}^{2}$ (top) and $e B=0.6 \mathrm{GeV}^{2}$ (bottom).

thinks about the quark masses instead, then we are looking at how the in-medium quark mass $M_{q}\left(T, \mu_{B}, e B\right)$ varies with respect to its magnetized vacuum value $M_{q}(0,0, e B)$.

The results for the normalized up-quark condensate $\langle u \bar{u}\rangle_{0}$ for both $G_{s}^{0}$ (constant coupling) and $G_{s}(e B)$ (magnetic coupling) models are in Fig. 1. The top panels show the results for $e B=0.3 \mathrm{GeV}^{2}$, while the bottom panels have the $e B=0.6 \mathrm{GeV}^{2}$ results. Furthermore, the left panels contain the $G_{s}^{0}$ model results, while the right panels display the $G_{s}(e B)$ model results. The following conclusions can be drawn. At low temperatures, the chiral transition between the broken and the (approximately) restored regions is of first order for all cases. The firstorder transition occurs at $\mu^{\text {crit }}(T)$, at which the condensate changes abruptly from $\langle u \bar{u}\rangle_{0}=1$ [or, looking at the quark mass, $\left.M_{u}(0,0, e B)\right]$, to the (approximately) restored chiral symmetry with a much lower value of $\langle u \bar{u}\rangle_{0}\left(M_{u} \approx m_{u}\right)$. Therefore, the chiral symmetry is restored via a strong (and unique) first-order phase transition from the vacuum value to an almost zero value of $\langle u \bar{u}\rangle_{0}$. The first-order transition persists in the phase diagram up to the CEP where the phase transition turns into second order. Above the CEP's temperature, the transition shows an analytic nature (crossover transition). The position of the CEP, $\left(T, \mu_{B}\right)_{\mathrm{CEP}}$, is given in Table I for each case. An important difference between the $G_{s}^{0}$ and $G_{s}(e B)$ models is clear: at zero temperature, the

TABLE I. The temperature, baryonic chemical potential, and baryonic density (in units of $\rho_{0}=0.16 \mathrm{fm}^{-3}$ ) at the light CEP, $\left(T, \mu_{B}, \rho_{B}\right)_{\mathrm{CEP}}$, for different values of $B\left(\right.$ in $\left.\mathrm{GeV}^{2}\right)$.

\begin{tabular}{llllllll}
\hline \hline & \multicolumn{3}{c}{$G_{s}=G_{s}^{0}$} & & \multicolumn{3}{c}{$G_{s}=G_{s}(e B)$} \\
\cline { 2 - 4 } \cline { 6 - 7 } CEP & $T(\mathrm{MeV}) \mu_{B}(\mathrm{MeV}) \rho_{B} / \rho_{0}$ & $T(\mathrm{MeV}) \mu_{B}(\mathrm{MeV}) \rho_{B} / \rho_{0}$ \\
\hline$e B=0$ & 157.5 & 890.4 & 1.74 & & 157.5 & 890.4 & 1.74 \\
$e B=0.3$ & 192 & 674 & 3.54 & & 177 & 627 & 2.63 \\
$e B=0.6$ & 214 & 692 & 7.22 & & 171 & 535 & 3.90 \\
\hline \hline
\end{tabular}



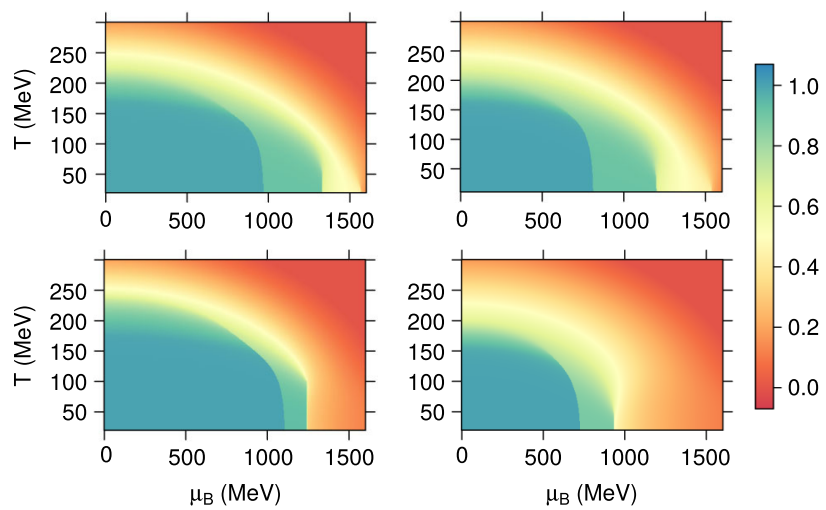

FIG. 2. The normalized strange-quark condensate $\langle s \bar{s}\rangle_{0}$ (the color scale represents its magnitude) with $G_{s}^{0}$ (left) and $G_{s}(e B)$ (right) for $e B=0.3 \mathrm{GeV}^{2}$ (top) and $e B=0.6 \mathrm{GeV}^{2}$ (bottom).

chemical potential at which the phase transition occurs, $\mu_{B}^{\text {crit }}(T=0)$, increases with $B$ for $G_{s}^{0}$, while the opposite happens for $G_{s}(e B)$. Likewise, the pseudocritical temperature at $\mu_{B}=0$ decreases for $G_{s}(e B)$ [as expected, due to the $G_{s}(e B)$ parametrization] and increases for $G_{s}^{0}$. Therefore, the overall effect of $G_{s}(e B)$ on the $\mu_{B}-T$ phase diagram is the diminishing of the region where chiral symmetry is broken.

The phase diagrams for the down quark, $\langle d \bar{d}\rangle_{0}$ $\left(T, \mu_{B}, e B\right)$, are similar to the up-quark results (Fig. 1) and are not shown. The main difference is that the crossover band, identified by the yellow band where $\langle d \bar{d}\rangle_{0} \approx 0.5$, which defines the pseudocritical transition temperature, is located at slightly lower temperatures for the down quark, due to the electric charge difference.

The phase diagrams for the normalized strange-quark condensate $\langle s \bar{s}\rangle_{0}$ are displayed in Fig. 2 (same configuration as in Fig. 1), showing the following interesting features. The condensate shows multiple discontinuities, indicating the presence of multiple first-order phase transitions, and thus the existence of multiple CEPs in the strange sector for all cases.

The existence of several first-order phase transitions at zero temperature in the presence of an external magnetic field for the SU(2) NJL model was reported in [49]. Due to the Landau quantization induced by the magnetic field, instead of a single first-order transition, connecting the vacuum phase to the chirally restored phase, several intermediate first-order phase transitions take place. These complex patterns of multiple phase transitions were analyzed at zero temperature in [50-52], where the number of first-order phase transitions, characterized by small jumps in the order parameters, were seen to grow as the magnetic field decreases. At zero temperature, each phase transition can be attributed to the filling of a specific Landau level. At finite temperature, even though all the Landau levels have a finite probability of being populated, at small temperatures, the multiple phase transitions can still be associated with the partial filling of the lower
Landau levels. With increasing temperature, the number of phase transitions decreases to just one, and thus the multiple CEPs that appear in the phase diagram (one for each first-order phase transition) decreases to just one. The several first-order phase transitions for the light sector, and the corresponding CEPs, were analyzed in [40]. In Fig. 1 just one first-order phase transition is present for the up quark (and down quark) because the magnetic fields considered are too high for multiple transitions to set in. For smaller magnetic fields, several phase transitions, and corresponding CEPs, are also present in the light sector (see [40]).

At $e B=0.3 \mathrm{GeV}^{2}$ (top panels of Fig. 2) both models show three first-order phase transitions that end up in three CEPs, while for $e B=0.6 \mathrm{GeV}^{2}$ (lower panels of Fig. 2) two first-order phase transitions are present. Let $\mu_{i}^{\text {crit }}(T)$ denote the chemical potential at which the first $(i=1)$, second $(i=2)$, and third $(i=3)$ first-order phase transitions take place, where $\mu_{1}^{\text {crit }}(T)<\mu_{2}^{\text {crit }}(T)<\mu_{3}^{\text {crit }}(T)$. The first first-order phase transition, $\mu_{1}^{\text {crit }}(T)$, at which $\langle s \bar{s}\rangle_{0}$ has a small jump, is induced on the strange quarks by the chiral transition of the light quarks (see Fig. 1). With the chiral symmetry already restored in the light sector for $\mu_{B}>\mu_{1}^{\text {crit }}(T)$, the two subsequent first-order phase transitions [i.e., $\mu_{2}^{\text {crit }}(T)$ and $\mu_{3}^{\text {crit }}(T)$ ], at which a sudden decrease of $\langle s \bar{s}\rangle_{0}$ occurs, can only be associated with the strange sector. Therefore, at lower temperatures, the strange quarks undergo a phase transition from a region of broken chiral symmetry to an (approximately) restored one via intermediate transitions. At $e B=0.3 \mathrm{GeV}^{2}$, the strange quark takes the following values: $\langle s \bar{s}\rangle_{0}$ for $\mu_{B}<\mu_{1}^{\text {crit }}(T)$, $0.9\langle s \bar{s}\rangle_{0} \quad$ for $\quad \mu_{1}^{\text {crit }}(T)<\mu_{B}<\mu_{2}^{\text {crit }}(T), \quad 0.5\langle s \bar{s}\rangle_{0}$ for $\mu_{2}^{\text {crit }}(T)<\mu_{B}<\mu_{3}^{\text {crit }}(T)$, and $0.1\langle s \bar{s}\rangle_{0}$ for $\mu_{B}$ slightly above $\mu_{3}^{\text {crit }}(T)$ and, then decreases smoothly with increasing $\mu_{B}$. The $G_{s}(e B)$ model predicts smaller values for $\mu_{1}^{\text {crit }}(T), \mu_{2}^{\text {crit }}(T)$, and $\mu_{3}^{\text {crit }}(T)$ than the $G_{s}^{0}$ model. At $e B=0.6 \mathrm{GeV}^{2}$, the same pattern occurs but now with just two phase transitions. The location of the bright band, which indicates the value $\left(T, \mu_{B}\right)$ where $\langle s \bar{s}\rangle_{0} \approx 0.5$, also shows that for $e B=0.3$, the first jump in the condensate at $\mu_{2}^{\text {crit }}(T)$ only reduces $\langle s \bar{s}\rangle_{0}$ slightly, and another second phase transition at $\mu_{3}^{\text {crit }}(T)$ is necessary to obtain a more complete restoration of the chiral symmetry. The positions of the CEPs are listed in Table II for each case.

The phase diagram for the confinement/deconfinement transition, determined by the Polyakov loop value, $\Phi\left(T, \mu_{B}, e B\right)$, is presented in Fig. 3. The deconfinement pseudocritical temperature $T^{\Phi}$, defined by $\Phi\left(T^{\Phi}, \mu_{B}\right)=0.5$, is a decreasing function of $\mu_{B}$ for all scenarios. The bright band represents a value of $\Phi \approx 0.5$, and thus it can be used as a visual guide of the pseudocritical deconfinement transition in the $T-\mu_{B}$ plane. The discontinuity in the Polyakov loop value only reflects the first-order chiral phase transition for the light sector. For small values of $\mu_{B}$, 
TABLE II. The temperature, baryonic chemical potential, and baryonic density (in units of $\rho_{0}=0.16 \mathrm{fm}^{-3}$ ) at the strange CEPs, $\left(T, \mu_{B}, \rho_{B}\right)_{\mathrm{CEP}}$, for different values of $B\left(\right.$ in $\left.\mathrm{GeV}^{2}\right)$.

\begin{tabular}{|c|c|c|c|c|c|c|}
\hline \multirow[b]{2}{*}{ CEP } & \multicolumn{3}{|c|}{$G_{s}=G_{s}^{0}$} & \multicolumn{3}{|c|}{$G_{s}=G_{s}(e B)$} \\
\hline & \multicolumn{6}{|c|}{$T(\mathrm{MeV}) \mu_{B}(\mathrm{MeV}) \rho_{B} / \rho_{0} T(\mathrm{MeV}) \mu_{B}(\mathrm{MeV}) \rho_{B} / \rho_{0}$} \\
\hline$e B=0$ & \multicolumn{3}{|c|}{ No CEP } & \multicolumn{3}{|c|}{ No CEP } \\
\hline \multirow{2}{*}{$e B=0.3$} & 6 & & & 48 & 1193 & 5.40 \\
\hline & & & & 18 & 1539 & 10.75 \\
\hline$e B=0.6$ & 124 & 1234 & 12.10 & 54 & 934 & 8.50 \\
\hline
\end{tabular}

the band of $\Phi \approx 0.5$ is very close to the crossover region of the light sector. For low temperatures and high chemical potential values, there is a quark phase on which the chiral phase is already restored but confinement is still realized (low $\Phi$ values). At $e B=0.6 \mathrm{GeV}^{2}$, the $G_{s}^{0}$ model (bottom right panel) presents an intersection of the Polyakov loop line $\Phi=0.5$ with the first-order chiral phase transition line in the vicinity of the CEP for the light quarks. As the magnetic field increases, an overlapping occurs between the first-order phase transition, which moves to higher temperatures for the $G_{s}^{0}$ model, and the deconfinement transition, which in turn remains almost unchanged by the magnetic field presence.

\section{B. Phase-separation boundaries}

In this section we briefly analyze the quark phase transitions through the phase-separation boundaries (binodals) and instability boundaries (spinodals).

We first consider the phase-separation boundaries at zero temperature in a $\mu_{B}-B$ plane. The results are in Fig. 4, where we display the spinodal lines (thick blue) and binodal line (thick black line) for the light quarks within both models. The spinodal region (blue area) increases with the magnetic field for both $G_{s}^{0}$ (left panel) and $G_{s}(e B)$ (right panel) models.

The pattern followed by the baryonic chemical potential value at which the light phase transition occurs at zero
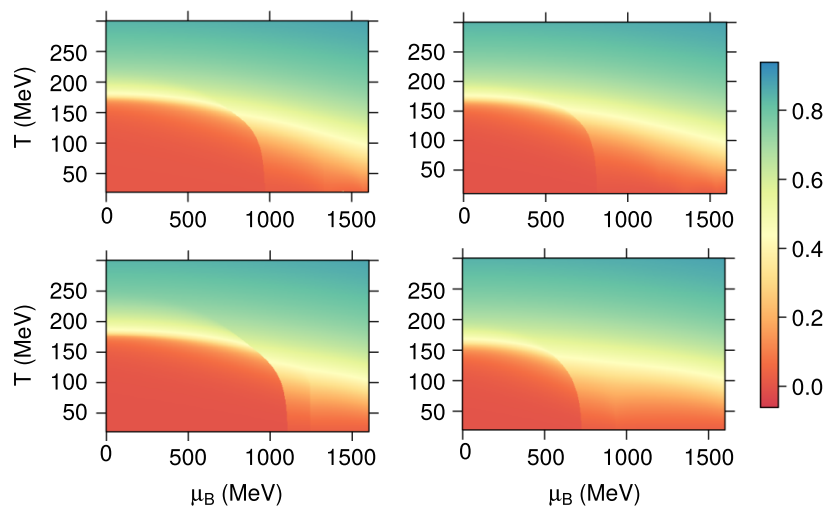

FIG. 3. The Polyakov loop (the color scale represents its magnitude) with $G_{s}^{0}$ (left) and $G_{s}(e B)$ (right) for $e B=$ $0.3 \mathrm{GeV}^{2}$ (top) and $e B=0.6 \mathrm{GeV}^{2}$ (bottom).

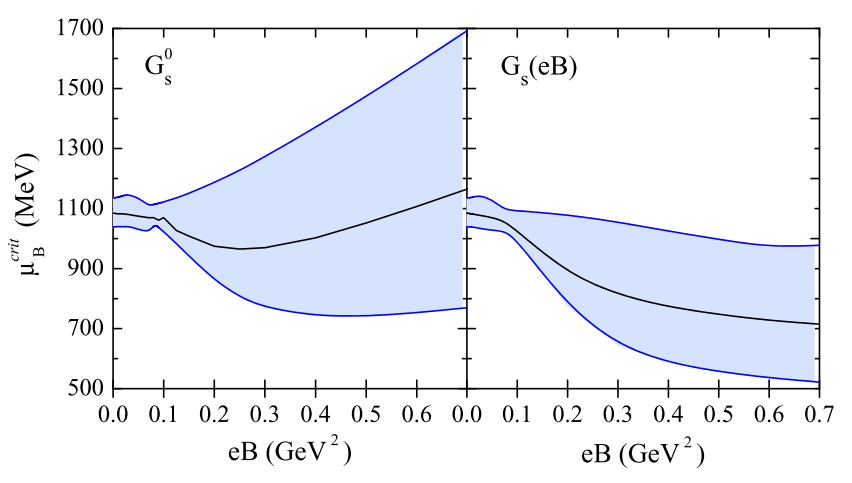

FIG. 4. The spinodal (blue thick line) and binodal (black thick line) $\mu_{B}^{\text {crit }}$ boundaries as a function of the magnetic field intensity at $T=0$. The models $G_{s}^{0}$ (left panel) and $G_{s}(e B)$ (right panel) are shown.

temperature, $\mu_{B}^{\text {crit }}(T=0)$, was studied in detail for the PNJL model in [43]. A lowering of $\mu_{B}^{\text {crit }}$ with $B$ was seen until $e B \approx 0.3 \mathrm{GeV}^{2}$ for $G_{s}^{0}$, followed by a monotonically increasing of the $\mu_{B}^{\text {crit }}$ for stronger field strengths. The $\mu_{B}^{\text {crit }}$ always decreases for the $G_{s}(e B)$ mode. The existence of a range of magnetic fields, where at least two first-order phase transitions occur for the light sector, was also pointed out. Therefore, instead of a single first-order transition, connecting the vacuum phase to the (approximately) chirally restored phase, we have multiple intermediate first-order phase transitions at $T=0$, that subsist at low and moderate temperatures, giving rise to multiple CEPs that appear in the phase diagram for the light sector $[40,43]$. Indeed, the magnetic field induces a complex pattern of phase transitions for all quarks, i.e., for both the light (up and down) and the strange quarks.

Another clear feature also from Fig. 4 is the spreading of the spinodal region in the $\mu_{B}$ direction with the increase of $B$ for both models, to a lower extent within the $G_{s}(e B)$ model. This has implications on the CEP location at finite temperatures, as seen in [43]. The temperature of the CEP is an increasing function of $B$ for the $G_{s}^{0}$ model, reflecting the increasing spreading of the spinodal region with $B$. The slower spreading of the spinodal region for the $G_{s}(e B)$ model, however, leads to an increase of the CEP's temperature only up to an intermediate $B$ strength. For higher $B$ fields, the width of the spinodal region remains approximately constant and the CEP's temperature remains almost unchanged (as we will see in Fig. 9).

In Figs. 5 and 6 we represent, respectively, $T-\mu_{B}$ and $T-\rho_{B}$ diagrams, where the binodals are represented by the thick lines and the spinodal by the thin lines. The phaseseparation boundaries are in blue for the light quarks and in green for the strange quark. Three magnetic field intensities are studied, 0 (top), 0.3 (middle), and $0.6 \mathrm{GeV}^{2}$ (bottom), for the $G_{s}^{0}$ (right) and $G_{s}(e B)$ (left) models. As already seen in Fig. 2, we have two CEPs for the strange sector at $e B=0.3 \mathrm{GeV}^{2}$. The existence of two CEPs for the strange 


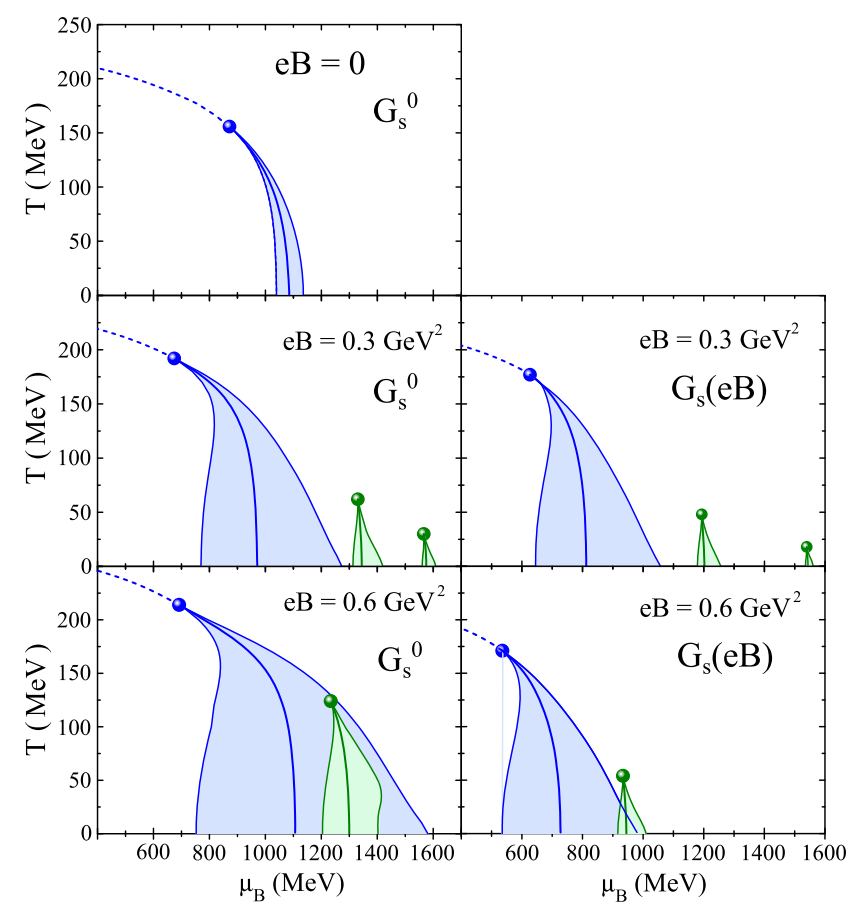

FIG. 5. Binodal (thick lines) and spinodal (thin lines) regions in the temperature vs baryonic chemical potential diagram for the light quarks (blue) and strange quarks (green) at 0 (top), 0.3 (middle), and $0.6 \mathrm{GeV}^{2}$. Both $G_{s}^{0}$ (left) and $G_{s}(e B)$ (right) model results are shown.

sector occurs, for both $G_{s}^{0}$ and $G_{s}(e B)$ scenarios, in the range $0.2 \gtrsim e B \gtrsim 0.4 \mathrm{GeV}^{2}$. For $e B \lesssim 0.2 \mathrm{GeV}^{2}$ more CEPs can exist due to the existence of numerous firstorder transitions, while above $0.4 \mathrm{GeV}^{2}$ only one CEP persists.

Another important aspect is that, the stronger the magnetic field is, the larger the spinodal region becomes for both models, being the spinodal region bigger when $G_{s}=G_{s}^{0}$. This is valid for both the light and strange transitions. The first-order lines are also shifted to lower values of $\mu_{B}$. For $e B=0.6 \mathrm{GeV}^{2}$ (lower panels of Fig. 5) the spinodal regions in the $\left(T, \mu_{B}\right)$ plane overlap with each other (blue for light quarks and green for strange quark). However, this happens at different baryonic densities (see lower panels of Fig. 6). The spinodal region for the strange quark is much smaller than for the light quarks and is located at higher baryonic densities.

From Fig. 6 at zero temperature, we conclude that the upper baryonic densities at which the onset of both spinodal and binodal regions take place are increasing functions of $B$.

\section{The location of the critical endpoints}

In this section, we determine the location of the CEPs in the temperature vs. baryonic chemical potential diagram, and its dependence on the magnetic field strength.

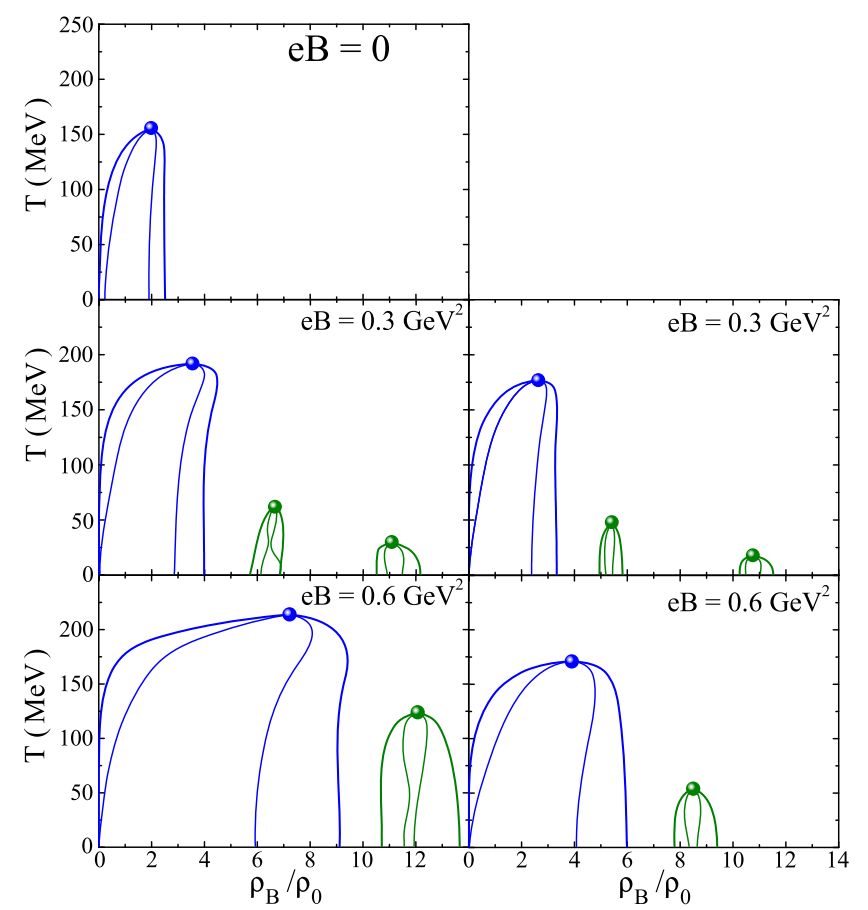

FIG. 6. Binodal curve (thick lines) and spinodal section (blue region) in the temperature vs. baryonic density diagram for the light quarks (blue) and strange quarks (green) at 0 (top), 0.3 (middle), and $0.6 \mathrm{GeV}^{2}$. Both $G_{s}^{0}$ (left) and $G_{s}(e B)$ (right) model results are shown $\left(\rho_{0}=0.16 \mathrm{fm}^{-3}\right)$.

Figure 7 shows the location of the CEPs as a function of the magnetic field, $\left(T(B), \mu_{B}(B)\right)_{\mathrm{CEP}}$. The behavior of the CEP related with the chiral (light) transition (blue and black) was already reported in [43]. For moderate magnetic fields $\left(<0.3 \mathrm{GeV}^{3}\right)$ both models, $G_{s}^{0}$ and $G_{s}(e B)$, show similar results; i.e., the CEP moves towards higher temperatures and chemical potentials. A distinctive behavior is

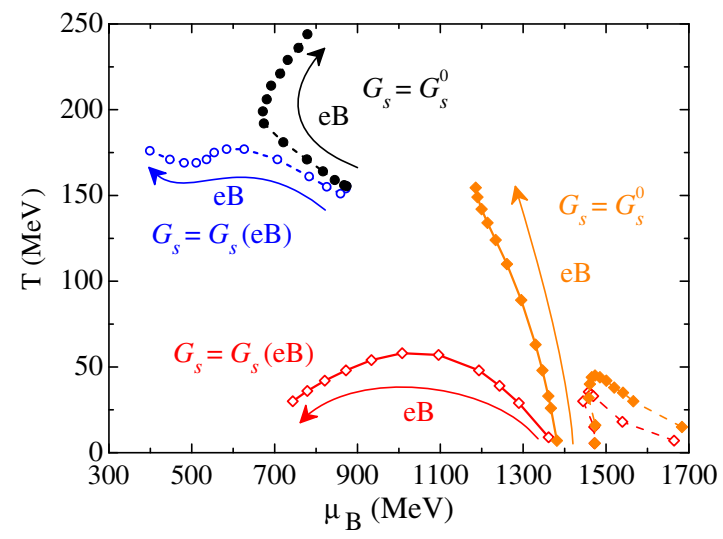

FIG. 7. Critical endpoint of the light (blue and black) and strange (red and orange) quarks as a function of the magnetic field intensity for the constant coupling, $G_{s}^{0}$, and magnetic dependent coupling, $G_{s}(e B)$, models. The magnetic field increases from 0 to $1 \mathrm{GeV}^{2}$ in the arrows' directions. 
seen for higher magnetic fields: the CEP moves to lower $\mu_{B}$ for the $G_{s}(e B)$ model, and the opposite occurs for the $G_{s}^{0}$ model. As already noticed in [43], the $G_{s}(e B)$ model results indicate that, for high enough magnetic fields, the CEP moves towards the $\mu_{B}=0$ axis, and the analytic transition, present at $\mu_{B}=0$, will turn into a first-order phase transition.

Now let us focus on the CEP for the strange quark. As we had already seen in Fig. 2 (bottom right panel), the magnetic field induces multiple first-order phase transitions for the strange quark, and thus the existence of multiple CEPs. In Fig. 7 is shown two CEP branches for each model [red for $G_{s}(e B)$ and orange for $G_{s}^{0}$ ]. For both models, the CEP appearing at lower $\mu_{B}$ remains up to $e B \sim 1 \mathrm{GeV}^{2}$, while the CEP at higher $\mu_{B}$ disappears from the phase diagram at $e B \sim 0.4 \mathrm{GeV}^{2}$ (a similar behavior was already found for the light sector [40]). The CEPs located at lower $\mu_{B}$ show a different behavior between models: while it moves towards lower $\mu_{B}$ in both models, at high magnetic fields $T$ increases monotonously with $B$ for $G_{s}^{0}$ and is a decreasing function for the $G_{s}(e B)$ model. With increasing $B$, the CEP's location for the $G_{s}(e B)$ model (red) shows some similarity with the CEP of the light quarks (blue) by moving to lower $\mu_{B}$. For the $G_{s}^{0}$ model (orange) the CEP goes to lower values of $\mu_{B}$ but higher $T$.

\section{The isentropic trajectories}

Finally, we analyze how the isentropic trajectories on the $T-\mu_{B}$ plane [53] and, in particular, near the CEPs are affected by the presence of an external magnetic field for both models, i.e., $G_{s}^{0}$ and $G_{s}(e B)$. The interest in the isentropic trajectories relies on the hydrodynamical expansion of a HIC fireball that nearly follows trajectories of constant entropy. New insights about the QCD phase diagram can thus be obtained by investigating these possible paths for the hydrodynamic evolution of a thermal medium created in the collisions and by studying the properties of matter under these conditions.

We plot in Fig. 8 several isentropic trajectories $s / \rho_{B}$ for both models and different magnetic field values on the $T-\mu_{B}$ plane. For the sake of comparability, the following scenarios have been selected in each panel: $e B=0$ (top left), $e B=0.3 \mathrm{GeV}^{2}$ for the $G_{s}^{0}$ model (top right), $e B=$ $0.3 \mathrm{GeV}^{2}$ for the $G_{s}(e B)$ model (bottom left), and finally
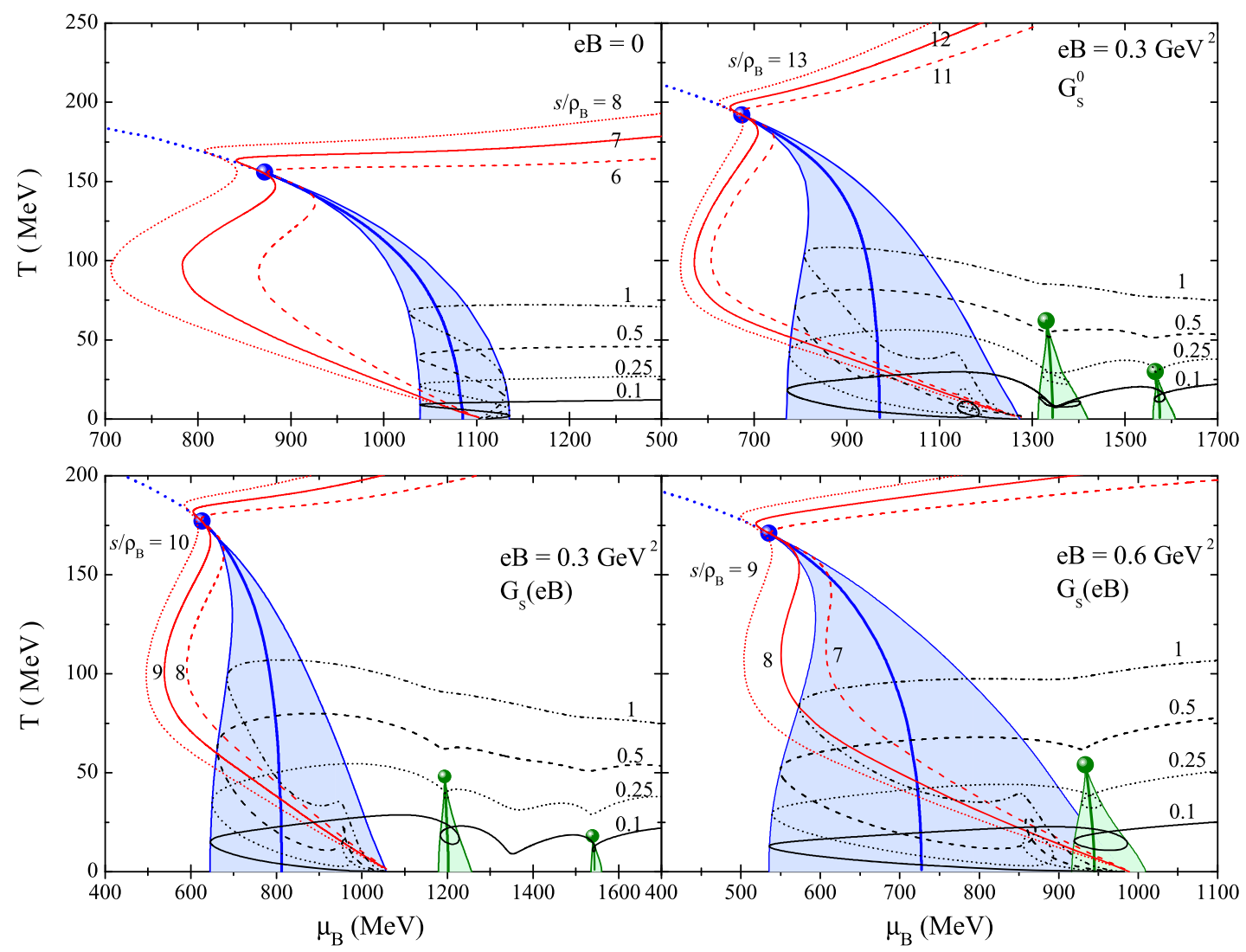

FIG. 8. QCD phase diagram in the $T$ - $\mu_{q}$ plane: the thick blue (green) lines represent the first-order phase transition (binodal) while the thin blue (green) lines display the spinodal boundaries for the light (strange) quarks. The isentropic trajectories for several values of $s / \rho_{B}$ are shown in red and black. The following scenarios are considered: the $G_{s}^{0}=G_{s}(e B)$ model for $e B=0$ (top left panel), the $G_{s}^{0}$ model for $e B=0.3 \mathrm{GeV}^{2}$ (top right panel), the $G_{s}(e B)$ model for $e B=0.3 \mathrm{GeV}^{2}$ (bottom left panel), and the $G_{s}(e B)$ model for $e B=0.6 \mathrm{GeV}^{2}$ (bottom right panel). The different scales in the $\mu_{B}$-axis allow us to clearly differentiate among the different isentropic trajectories. 
$e B=0.6 \mathrm{GeV}^{2}$ for the $G_{s}(e B)$ model (bottom right). For a clear distinction among the different isentropic trajectories, we have restricted the study to the range of $\mu_{B}$ values on which the binodals (metastable boundaries) occur in each case. From all possible isentropic lines (constant $s / \rho_{B}$ ) we have restricted ourselves to two sets: (i) trajectories with higher values of $s / \rho_{B}$ represented by red lines that pass close to the CEP of the light quarks; (ii) trajectories with $s / \rho_{B} \leq 1$ displayed in black lines that go through the firstorder phase transition line and, at larger $\mu_{B}$, pass near the CEPs connected with the strange sector.

In the following we discuss the behavior of the isentropic lines as temperature increases. At zero temperature, all isentropic trajectories begin at the same $\mu_{B}$ value, i.e., $\mu_{i}=M_{i}$, which increases with $B$ (see Fig. 8). The temperature of the isentropic path $s / \rho_{B}$ takes a finite value as soon as $\rho_{B}$ becomes finite.

For low values of the entropy per particle, a special pattern arises among the calculated isentropic trajectories (black lines): they are enclosed within the spinodal boundary, which in the $T-\rho$ plane encloses the unstable and metastable regions limited by the binodals. Eventually, as the chemical potential further increases, the isentropic line leaves this region and proceeds towards the high $\mu_{B}$ chiral restored phase, keeping the temperatures approximately constant for $B=0$, but showing a decrease, or eventually, for the lower $s / \rho_{B}$ values, still an increase followed by a decrease for $e B=0.3,0.6 \mathrm{GeV}^{2}$. As we will discuss later, this is due to the onset of the strange quark, that is pushed to lower values of $\mu_{B}$ and $\rho_{B}$ at finite $B$.

For higher $s / \rho_{B}$ values (red lines), however, as the temperature increases from $T=0$ the trajectories cross the spinodal region entering the stable low density and chiral broken phase, moving towards the CEP, where a kink occurs in the $T-\mu_{B}$ plane, and then move to higher $\mu_{B}$ (chiral restored phase) always with increasing temperature. As we will discuss later, this kink is not present in the $T-\rho_{B}$ plan (see Fig. 9) and is a feature of the presence of the CEP in the $T-\mu_{B}$ plane.

A different and interesting aspect is the trajectories' behavior near the strange quark CEPs, to which they are attracted. Even though there is no focusing effect on the
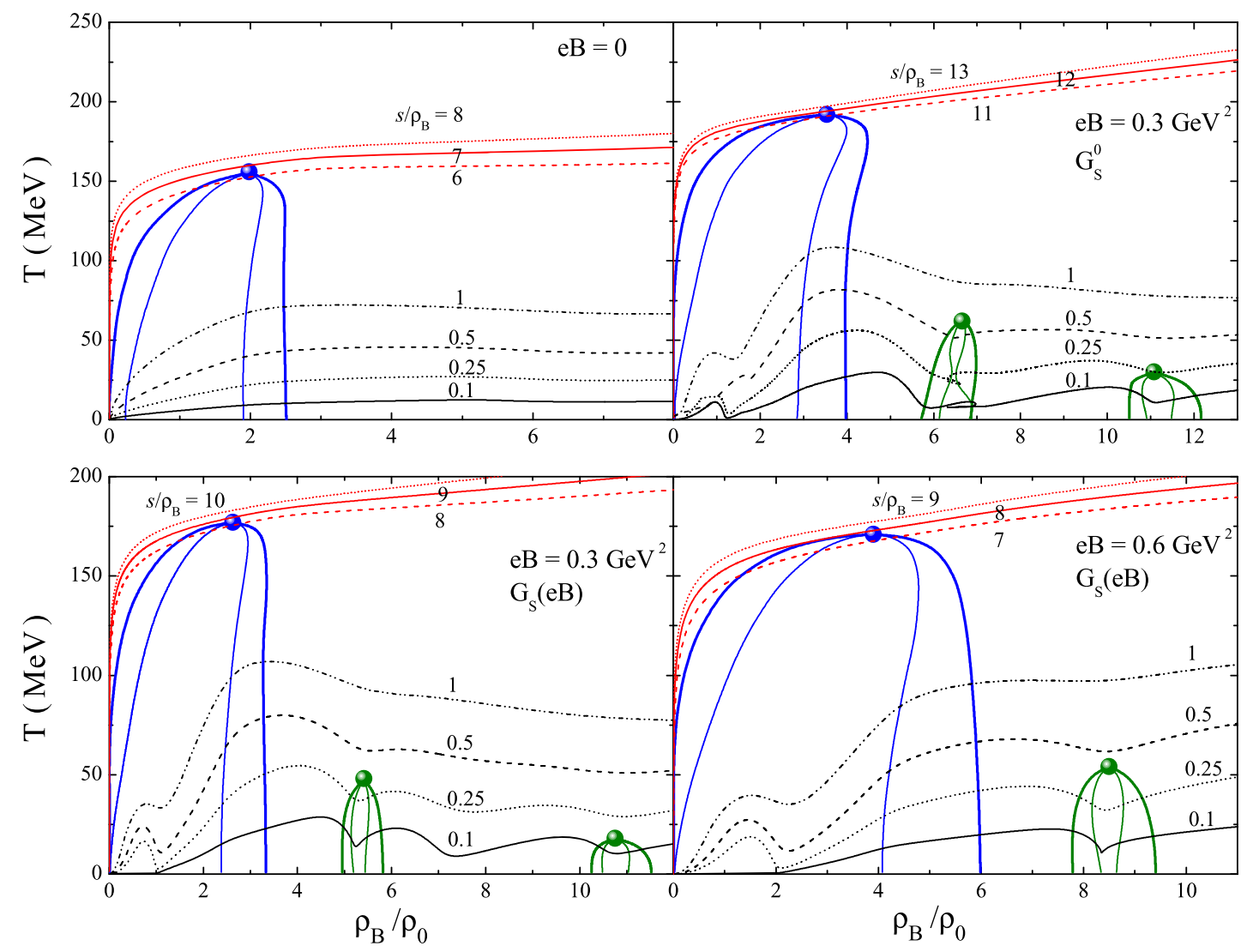

FIG. 9. QCD phase diagram in the $T-\rho_{B}$ plane: the thick blue (green) lines represent binodal boundaries, while the thin blue (green) lines display the spinodal boundaries for the light (strange) quarks. The isentropic trajectories for several values of $s / \rho_{B}$ are shown in red and black. The following scenarios are considered: the $G_{s}^{0}=G_{s}(e B)$ model for $e B=0$ (top left panel), the $G_{s}^{0}$ model for $e B=$ $0.3 \mathrm{GeV}^{2}$ (top right panel), the $G_{s}(e B)$ model for $e B=0.3 \mathrm{GeV}^{2}$ (bottom left panel), and the $G_{s}(e B)$ model for $e B=0.6 \mathrm{GeV}^{2}$ (bottom right panel). The different scales in the $\rho_{B}$-axis allow us to clearly differentiate among the different isentropic trajectories. The baryonic density $\rho_{B}$ is represented in units of saturation density, $\rho_{0}=0.16 \mathrm{fm}^{3}$. 
isentropic trajectories towards the CEP for the light quarks (see the red curves in Fig. 8), the CEPs related with the strange quark show a contrasting effect. This behavior allows the prediction of other new CEPs if lower values of $B$ are analyzed: looking at the bottom left panel of Fig. 8, the bend present near $\mu_{B}=1350 \mathrm{MeV}$ signals that a CEP would emerge if we decrease the magnetic field strength. The isentropes are quite affected by the growth of the spinodal region (related with the strengthening of the firstorder transition due to the magnetic field), particularly for the light sector, and are pushed to higher $T$ in the transition region. The explanation for this behavior will be more clearly discussed looking to the phase diagram in a $T-\rho_{B}$ plane as will be done in the following.

Finally, the shape of the isentropes also allows the perception of the spinodal region. Taking the line with $s / \rho_{B}=1$ for $e B=0$ at the lower temperatures (upper left panel) we see that this isentropic is bound by the spinodal lines of the light sector. For finite $B$ the same effect is present. However, looking at Fig. 8, we find a loop structure for the $s / \rho_{B}=0.1$ and $s / \rho_{B}=0.25$ lines inside the spinodal region for the light sector (blue region). This is not related with the existence of a second first-order transition for the light sector but with the onset of the up quark (as we will see in Fig. 10).

Additional insight can be attained by analyzing the $T-\rho_{B}$ diagrams. The results are shown in Figs. 9 and 10 (with the same configuration of Fig. 8). These phase diagrams show the density range of both unstable and metastable regions. In Fig. 9, the behavior of the trajectories with higher $s / \rho_{B}$ values (red lines) agrees for all scenarios: in the range of lower $\rho_{B}$ values, the required entropy per baryon is accomplished by a sudden increase of temperature. For $s / \rho_{B} \leq 1$ (black lines) and $B \neq 0$, the isentropic trajectories show a nonmonotonic behavior in the $T-\rho_{B}$ plane. This can be understood as follows: the entropy abruptly increases when new degrees of freedom appear, such as a new quark species. Thus, to keep $s / \rho_{B}$ fixed, a suddenly decrease of temperature is needed to compensate this abrupt increase in entropy. This can be seen in Fig. 10, where we have plotted the isentropic line with $s / \rho_{B}=0.25$, the quark masses $\left(M_{i}\right.$ with full colored lines), and quark densities ( $\rho_{i}$ with dashed
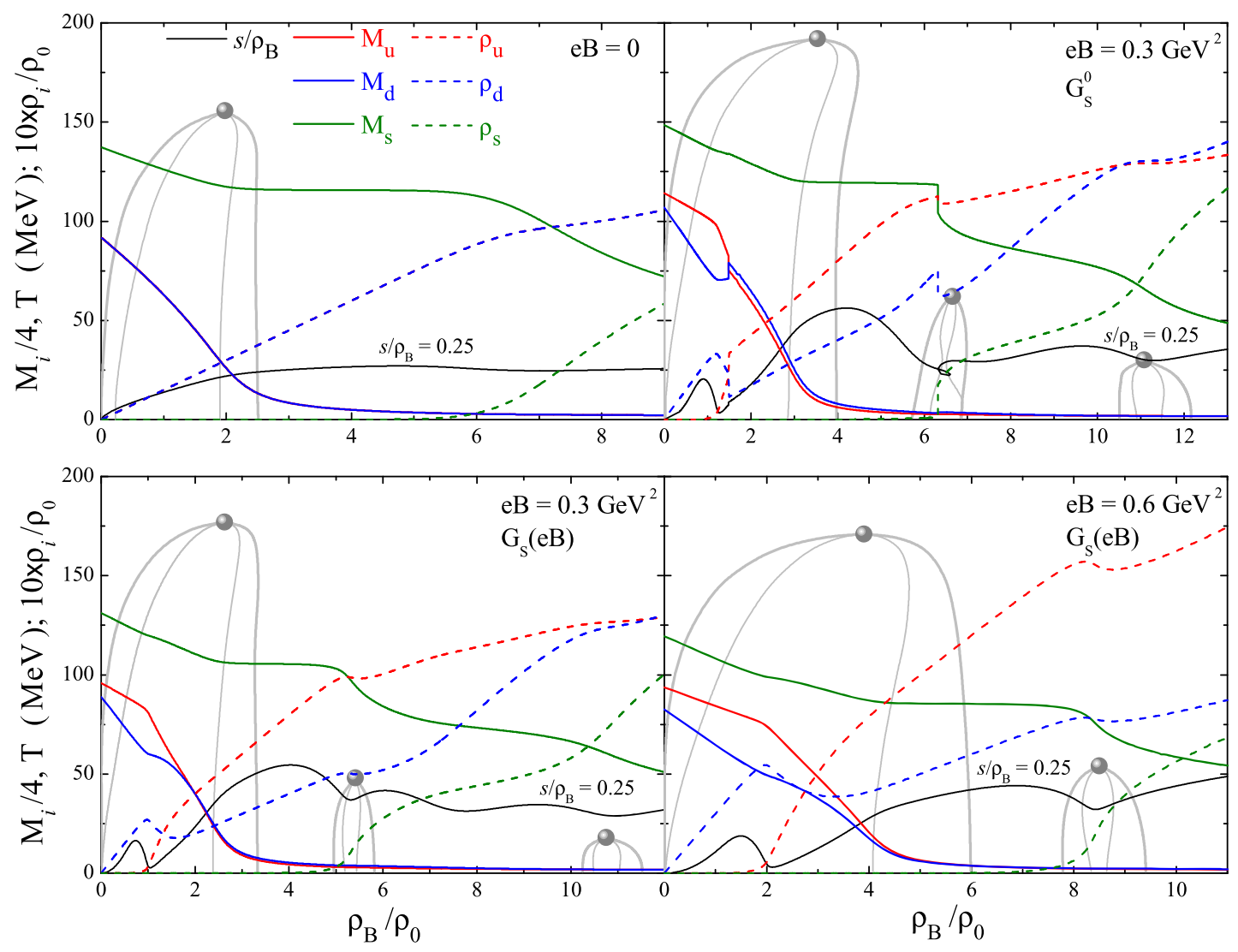

FIG. 10. QCD phase diagram in the $T-\rho_{B}$ plane: the thick gray lines represent the binodal boundaries, while the thin gray lines display the spinodal boundaries for the light and strange quarks. The isentropic trajectory for $s / \rho_{B}=0.25$ is shown as a black solid line. The masses (solid lines) and densities (dashed lines) for each quark (up in red, down in blue, and strange in green) are also displayed. The following scenarios are considered: the $G_{s}^{0}=G_{s}(e B)$ model for $e B=0$ (top left panel), the $G_{s}^{0}$ model for $e B=0.3 \mathrm{GeV}^{2}$ (top right panel), the $G_{s}(e B)$ model for $e B=0.3 \mathrm{GeV}^{2}$ (bottom left panel), and the $G_{s}(e B)$ model for $e B=0.6 \mathrm{GeV}^{2}$ (bottom right panel). The different scales in the $\rho_{B}$-axis allow us to clearly differentiate among the different isentropic trajectories. The baryonic density $\rho_{B}$ is represented in units of saturation density, $\rho_{0}=0.16 \mathrm{fm}^{3}$. 
lines) for all scenarios. For instance, looking at the $G_{s}(e B)$ model results for $e B=0.6 \mathrm{GeV}^{2}$ (right bottom panel), we conclude that in the range of densities $\rho_{B} \approx 1.5 \rho_{0}-2.2 \rho_{0}$ the temperature of the isentropic line decreases, and that the temperature also decreases at $\rho_{B} \approx 7.0 \rho_{0}$. The reason is because at $\rho_{B} \approx 1.5 \rho_{0}$ the density of the up quark becomes finite and at $\rho_{B} \approx 7.0 \rho_{0}$ the strange quark density takes a nonzero value. The same pattern is present for all scenarios. Other less dramatic effects are related with the partial restoration of the chiral symmetry of the strange quark that occurs in several steps: a further decrease of $M_{s}$ gives rise to an increase of the strange quark density, and therefore a more equal distribution of $\rho_{B}$ among all quark flavors, but consequently a decrease of $T$ to keep $s / \rho_{B}$ constant.

The change of the properties of matter along the isentropes in the presence of a strong magnetic field will give rise to signatures of $B$ that could be identified. These could be (a) a much higher abundance of $\pi^{0}$ pions at low densities than the corresponding charged pions due to the late onset of the $u$-quarks or (b) the detection of a large amount of strange mesons. These features, however, require special matter conditions obtained from the HIC, namely, large densities and moderate temperatures.

\section{CONCLUSIONS}

We have studied the magnetized phase diagram for $(2+1)$-flavor quark matter within the PNJL model. Besides the usual PNJL model with constant scalar coupling, we have also considered a magnetic field dependent coupling, which reproduces the IMC effect at $\mu_{B}=0$.

The computed phase diagrams show that the nature of the deconfinement transition is quite insensitive to the external magnetic field strength for both models, preserving the analytic nature throughout the phase diagram. The quark condensates show, however, a distinct behavior between models. For the light quarks, the constant scalar coupling model gives rise to a region of broken chiral symmetry that increases with $B$, while for the magnetic field dependent coupling model it decreases with $B$. The strange quark shows multiple first-order phase transitions at low temperatures, giving rise to multiple CEPs on the phase diagram. Therefore, the chiral symmetry on the strange sector is partially restored through multiple phase transitions. The magnetic field induces a complex pattern of phase transitions not only for the strange quark, but also for the light quarks. At higher temperatures, the strange quark undergoes an analytic transition whose behavior and location are weakly model dependent.
We have analyzed the quark phase transitions through the phase-separation boundaries (binodals) and instability boundaries (spinodals). For all flavors and within both models, the results show that the spinodal region grows with increasing $B$. However, the spinodal section associated with the strange quark is smaller and is located at higher baryonic densities.

We have studied how the multiple CEPs' locations vary when the magnetic field strength is increased. Due to the occurrence of multiple first-order phase transitions, in both light and strange quarks, multiple CEPs emerge in the phase diagram. For the strange quark, we have calculated the location of the two CEPs that appear at lower $\mu_{B}$ values. While the first CEP (at lower $\mu_{B}$ ) remains in the phase diagram up to $e B \sim 1 \mathrm{GeV}^{2}$, the second CEP (at higher $\mu_{B}$ ) disappears at $e B \sim 0.4 \mathrm{GeV}^{2}$. The location of the first CEP depends on the model: while, at lower $B$, it moves towards lower $\mu_{B}$ values in both models, at higher $B$ it increases monotonically with $B$ for $G_{s}^{0}$ and decreases for $G_{s}(e B)$.

The isentropic trajectories in the $T-\mu_{B}$ and $T-\rho_{B}$ planes for both models and magnetic fields were calculated. The isentropes are affected by the growth of the spinodal region, particularly for the light sector, and are pushed to higher temperatures in the transition region, for large values of the entropy per baryon. Among the calculated values, it was shown that the temperature along the isentropic lines for $s / \rho_{B} \leq 1$ clearly indicates the appearance of new degrees of freedom or the (partial) restoration of chiral symmetry by decreasing with $\rho_{B}$ instead of increasing as at $B=0$. It is expected that the production of mesons during the HIC reflects the composition of matter at a given density and, therefore, may act as signatures of the presence of an intense magnetic field. The CEPs related to the strange quark transitions show a focusing effect, which is explained by the appearance of strangeness in matter. The focusing effect that occurs at larger densities was attributed to the partial restoration of the chiral symmetry for the strange quark.

\section{ACKNOWLEDGMENTS}

This work was supported by "Fundação para a Ciência e Tecnologia," Portugal, under the Project No. UID/FIS/ 04564/2016, under the Grant No. SFRH/BPD/102273/ 2014 (P.C.), and under the Project No. CENTRO-010145-FEDER-000014 (M.F.) through the CENTRO 2020 program. This work was partly supported by "NewCompstar," COST Action MP1304. 
[1] Z. Fodor and S. D. Katz, J. High Energy Phys. 04 (2004) 050.

[2] P. de Forcrand and O. Philipsen, Nucl. Phys. B673, 170 (2003).

[3] C. S. Fischer, J. Luecker, and C. A. Welzbacher, Phys. Rev. D 90, 034022 (2014).

[4] P. Costa, M. C. Ruivo, and C. A. de Sousa, Phys. Rev. D 77, 096001 (2008); P. Costa, C. A. de Sousa, M. C. Ruivo, and Y. L. Kalinovsky, Phys. Lett. B 647, 431 (2007).

[5] J. Moreira, J. Morais, B. Hiller, A. A. Osipov, and A. H. Blin, Phys. Rev. D 91, 116003 (2015).

[6] P. Costa, C. A. de Sousa, M. C. Ruivo, and H. Hansen, Europhys. Lett. 86, 31001 (2009); P. Costa, M. C. Ruivo, C. A. de Sousa, and H. Hansen, Symmetry 2, 1338 (2010).

[7] M. Bluhm, B. Kampfer, R. Schulze, D. Seipt, and U. Heinz, Phys. Rev. C 76, 034901 (2007).

[8] C. DeTar, L. Levkova, S. Gottlieb, U. M. Heller, J. E. Hetrick, R. Sugar, and D. Toussaint, Phys. Rev. D 81, 114504 (2010).

[9] S. Borsanyi, G. Endrodi, Z. Fodor, S. D. Katz, S. Krieg, C. Ratti, and K. K. Szabo, J. High Energy Phys. 08 (2012) 053.

[10] M. Asakawa, S. A. Bass, B. Muller, and C. Nonaka, Phys. Rev. Lett. 101, 122302 (2008).

[11] P. Senger et al., The CBM Physics Book, edited by B. Friman, C. Höhne, J. Knoll, S. Leupold, J. Randrup, R. Rapp, and P. Senger, Lect. Notes Phys. vol. 814 (Springer, Berlin, 2011), p. 681.

[12] B. I. Abelev et al. (STAR Collaboration), Phys. Rev. C 81, 024911 (2010).

[13] L. Adamczyk et al. (STAR Collaboration), Phys. Rev. Lett. 113, 092301 (2014).

[14] L. Adamczyk et al. (STAR Collaboration), arXiv: 1709.00773 .

[15] L. Adamczyk et al. (STAR Collaboration), Phys. Rev. Lett. 112, 032302 (2014).

[16] K. Grebieszkow (NA61/SHINE Collaboration), arXiv: 1709.10397.

[17] A. Aduszkiewicz (NA61/SHINE Collaboration), Nucl. Phys. A967, 35 (2017).

[18] Y. Akiba et al., arXiv:1502.02730.

[19] F. Bruckmann, G. Endrödi, and T. G. Kovacs, J. High Energy Phys. 04 (2013) 112.

[20] G. S. Bali, F. Bruckmann, G. Endrödi, Z. Fodor, S. D. Katz, S. Krieg, A. Schäfer, and K. K. Szabó, J. High Energy Phys. 02 (2012) 044.

[21] G. S. Bali, F. Bruckmann, G. Endrödi, Z. Fodor, S. D. Katz, and A. Schäfer, Phys. Rev. D 86, 071502 (2012).

[22] E.-M. Ilgenfritz, M. Muller-Preussker, B. Petersson, and A. Schreiber, Phys. Rev. D 89, 054512 (2014).

[23] M. D'Elia, S. Mukherjee, and F. Sanfilippo, Phys. Rev. D 82, 051501 (2010).

[24] V. G. Bornyakov, P. V. Buividovich, N. Cundy, O. A. Kochetkov, and A. Schäfer, Phys. Rev. D 90, 034501 (2014).

[25] V. A. Miransky and I. A. Shovkovy, Phys. Rep. 576, 1 (2015).

[26] J. O. Andersen, W. R. Naylor, and A. Tranberg, Rev. Mod. Phys. 88, 025001 (2016).
[27] K. Fukushima and Y. Hidaka, Phys. Rev. Lett. 110, 031601 (2013).

[28] J. Chao, P. Chu, and M. Huang, Phys. Rev. D 88, 054009 (2013).

[29] M. Ferreira, P. Costa, D. P. Menezes, C. Providência, and N. N. Scoccola, Phys. Rev. D 89, 016002 (2014).

[30] M. Ferreira, P. Costa, and C. Providência, Phys. Rev. D 89, 036006 (2014).

[31] M. Ferreira, P. Costa, O. Lourenço, T. Frederico, and C. Providência, Phys. Rev. D 89, 116011 (2014).

[32] R. L. S. Farias, K. P. Gomes, G. I. Krein, and M. B. Pinto, Phys. Rev. C 90, 025203 (2014).

[33] M. Ferreira, P. Costa, and C. Providência, Phys. Rev. D 90, 016012 (2014).

[34] A. Ayala, M. Loewe, A. J. Mizher, and R. Zamora, Phys. Rev. D 90, 036001 (2014).

[35] A. Ayala, M. Loewe, and R. Zamora, Phys. Rev. D 91, 016002 (2015).

[36] A. Ayala, C. A. Dominguez, L. A. Hernandez, M. Loewe, and R. Zamora, Phys. Rev. D 92, 096011 (2015); 92, 119905 (2015).

[37] A. Ayala, C. A. Dominguez, L. A. Hernandez, M. Loewe, and R. Zamora, Phys. Lett. B 759, 99 (2016).

[38] A. Ayala, C. A. Dominguez, L. A. Hernandez, M. Loewe, A. Raya, J. C. Rojas, and C. Villavicencio, Phys. Rev. D 94, 054019 (2016).

[39] V. P. Pagura, D. Gomez Dumm, S. Noguera, and N. N. Scoccola, Phys. Rev. D 95, 034013 (2017).

[40] P. Costa, M. Ferreira, H. Hansen, D. P. Menezes, and C. Providência, Phys. Rev. D 89, 056013 (2014).

[41] S. S. Avancini, D. P. Menezes, M. B. Pinto, and C. Providencia, Phys. Rev. D 85, 091901 (2012).

[42] M. Ruggieri, L. Oliva, P. Castorina, R. Gatto, and V. Greco, Phys. Lett. B 734, 255 (2014).

[43] P. Costa, M. Ferreira, D. P. Menezes, J. Moreira, and C. Providência, Phys. Rev. D 92, 036012 (2015).

[44] T. Hatsuda and T. Kunihiro, Phys. Rep. 247, 221 (1994).

[45] S. P. Klevansky, Rev. Mod. Phys. 64, 649 (1992).

[46] S. Roessner, C. Ratti, and W. Weise, Phys. Rev. D 75, 034007 (2007).

[47] P. Rehberg, S. P. Klevansky, and J. Hufner, Phys. Rev. C 53, 410 (1996).

[48] D. P. Menezes, M. B. Pinto, S. S. Avancini, A. Perez Martinez, and C. Providência, Phys. Rev. C 79, 035807 (2009); D. P. Menezes, M. B. Pinto, S. S. Avancini, and C. Providência, Phys. Rev. C 80, 065805 (2009).

[49] D. Ebert, K. G. Klimenko, M. A. Vdovichenko, and A. S. Vshivtsev, Phys. Rev. D 61, 025005 (1999).

[50] P. G. Allen and N. N. Scoccola, Phys. Rev. D 88, 094005 (2013).

[51] R. Z. Denke and M. B. Pinto, Phys. Rev. D 88, 056008 (2013).

[52] A. G. Grunfeld, D. P. Menezes, M. B. Pinto, and N. N. Scoccola, Phys. Rev. D 90, 044024 (2014).

[53] P. Costa, Phys. Rev. D 93, 114035 (2016). 